\title{
COMMENT
}

\section{Reply to the comment by Bochdansky \& Deibel}

\author{
Erica J. H. Head*
}

Ocean Sciences Division, Bediord Institute of Oceanography, PO Box 1006, Dartmouth, Nova Scotia, Canada B2Y 4A2

Bochdansky \& Deibel have examined the data from Figs. 4 \& 7 of Head \& Harris (1996) (H\&H), and have criticised it for the following reasons: firstly, they have suggested that Fig. 7 represents an 'autocorrelation', because the $y$-variable was derived by multiplying the $x$-and $y$-variables from Fig. 4, and this was then plotted against $x$ in Fig. 7; and secondly, they have suggested that the data in Fig. 7 can be better fitted to a Michaelis-Menten function, than by our linear formulation. We would argue that both of their suggestions are inappropriate. In the case of the derivation of the data in Fig. 7 from the data in Fig. 4, it should be noted that the $x$-and $y$-variables in Fig. 4 are not independent. The $y$-variable is ( $\Delta$ chla $-\Delta$ phaeo $) \times 100 /$ chla and the $x$-variable is effectively $\Delta$ chla/ $\Delta t$. Thus, when $x$ and $y$ are multiplied together (and divided by 100), they give $(\Delta \mathrm{chl} a-\Delta$ phaeo) $/ t$. Thus, what we are plotting in Fig. 7 is actually ( $\Delta$ chla $-\Delta$ phaeo) $/ t$ versus $\Delta c h l a / t$. This is not equivalent to plotting $x y$ versus $x$. The way we arrived at the original formulation was by logic, which is why it was written that way in the text, but there is no 'autocorrelation' in the sense that these authors have suggested. When it comes to fitting a Michaelis-Menten rather than a linear function to the data in Fig. 7, we cannot argue that the $r^{2}$ might not be higher, but as we show in the accompanying figure (Fig. 1B), the replot of Fig, 4 using the MichaelisMenten parameters provided by Bochdansky \& Deibel simply does not fit the observations, i.e. pigment destruction does not approach $100 \%$ as ingestion rates become very low. Our linear function replotted as in Fig. 4 gives a much better representation of the experimental observations.

We should also add to this discussion that as we stated in the original paper, our mathematical repre-

•E-mail: erica.head@maritimes.dfo.ca sentation was simplistic at best. Fitting a MichaelisMenten function might have seemed appropriate, because we are looking at an enzyme activity, but it should be noted that the $x$-variable in Fig. 7 is not
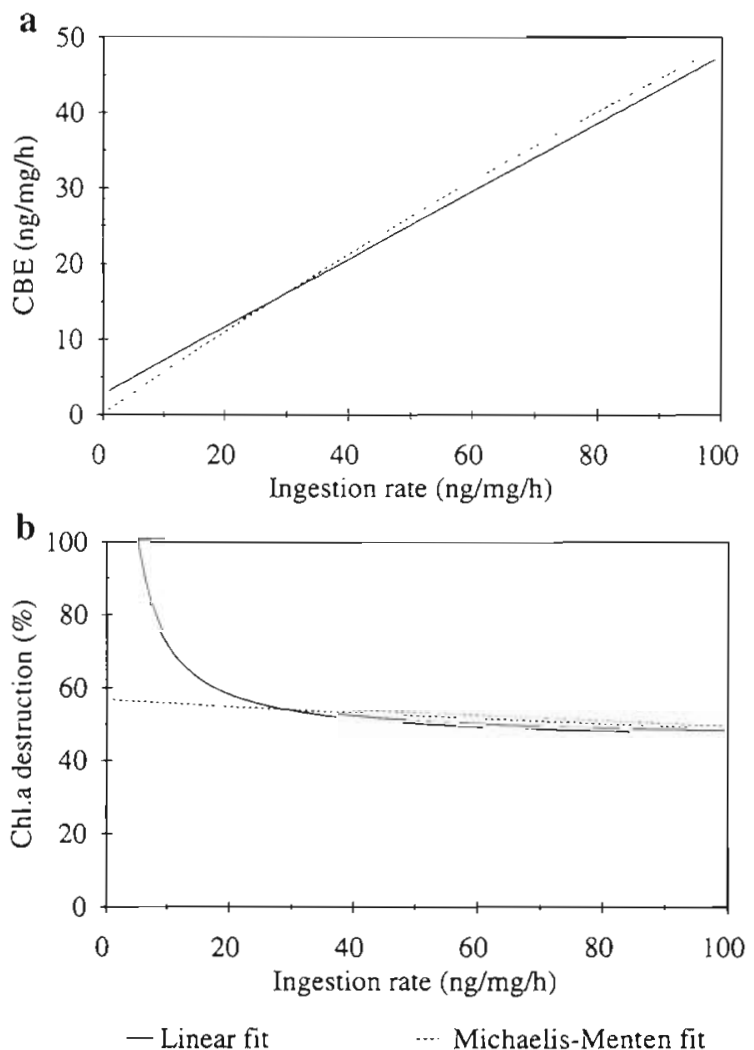

Fig. 1 (a) Comparison of the linear regression model of Head \& Harris (1996) and the Michaelis-Menten saturation equation proposed by Bochdansky \& Deibel. CBE: chlorophyll bleaching enzyme activity. (b) Comparison of the estimates of chlorophyll destruction versus ingestion rate derived from the linear regression of Head \& Harris (1996) or the MichaelisMenten equation of Bochdansky \& Deibel 
substrate concentration, but substrate uptake rate. The relationship between the measured ingestion rate and the concentration of substrate in the appropriate 'compartment' within the copepod is not necessarily straightforward: the substrate (chla) is ingested as a protein complex, within a chloroplast, within a phytoplankton cell. Furthermore, chla can be broken down by either of 2 competing enzymatic pathways: one which leads to colourless products and one which leads to phaeopigments. Thus, a simple MichaelisMenten function should not even apply.

Bochdansky \& Deibel have also suggested that the pattern of enzyme activity versus ingestion rate shown in $\mathrm{H} \& \mathrm{H}$ Fig. 7 is compatible with the enzyme being derived only from the copepod, instead of its activity being a combination of activities derived from both copepod and phytoplankton, as suggested by $\mathrm{H} \& \mathrm{H}$. We suggest that regardless of Fig. 7, our suggestion concerning the source of the enzyme could be argued based simply on experimental observations: firstly, it is easy to demonstrate that homogenates of many diatoms destroy chla (E. Head unpubl. data) and there is no evidence that the internal $\mathrm{pH}$ of copepods would destroy ingested enzymes immediately (e.g. Pond et al. 1995); and, secondly, the destruction apparently occurs very rapidly, perhaps even in the fore-gut. This being the case it is difficult to see how the copepods' capacity for chla destruction (CBE) could be increased quickly enough to destroy a relatively large proportion of ingested chla (either by de novo synthesis or secretion from pre-stored pools) when ingestion rates are high.

Acknowledgements. The author thanks Dr S. Sathyendranath for her participation in helpful discussions during the preparation of the manuscript

\section{LITERATURE CITED}

Head EJH, Harris LR (1996) Chlorophyll destruction by Calanus spp. grazing on phytoplankton: kinetics, effects of ingestion rate and feeding history, and a mechanistic. interpretation. Mar Ecol Prog Ser 135:223-235

Pond DW, Harris RP, Brownlee C (1995) A microinjection technique using a $\mathrm{pH}$-sensitive dye to determine the gut $\mathrm{pH}$ of Calanus helgolandicus. Mar Biol 123:75-79 